\title{
Impact of insufficient sleep on dysregulated blood glucose control under standardised meal conditions
}

\author{
Neli Tsereteli ${ }^{1}$ - Raphael Vallat ${ }^{2}$ • Juan Fernandez-Tajes ${ }^{1} \cdot$ Linda M. Delahanty ${ }^{3}$. Jose M. Ordovas ${ }^{4,5}$ • David A. Drew ${ }^{6,7}$. \\ Ana M. Valdes ${ }^{8}$. Nicola Segata ${ }^{9,10}$. Andrew T. Chan ${ }^{6,7}$ • Jonathan Wolf ${ }^{11}$ - Sarah E. Berry ${ }^{12}$. Matthew P. Walker ${ }^{2}$. \\ Timothy D. Spector ${ }^{13} \cdot$ Paul W. Franks ${ }^{1,14}$ iD
}

Received: 23 April 2021 / Accepted: 3 August 2021 / Published online: 30 November 2021

(C) The Author(s) 2021

\begin{abstract}
Aims/hypothesis Sleep, diet and exercise are fundamental to metabolic homeostasis. In this secondary analysis of a repeated measures, nutritional intervention study, we tested whether an individual's sleep quality, duration and timing impact glycaemic response to a breakfast meal the following morning.

Methods Healthy adults' data $(N=953$ [41\% twins]) were analysed from the PREDICT dietary intervention trial. Participants consumed isoenergetic standardised meals over 2 weeks in the clinic and at home. Actigraphy was used to assess sleep variables (duration, efficiency, timing) and continuous glucose monitors were used to measure glycaemic variation ( $>8000$ meals).

Results Sleep variables were significantly associated with postprandial glycaemic control ( $2 \mathrm{~h}$ incremental AUC), at both between- and within-person levels. Sleep period time interacted with meal type, with a smaller effect of poor sleep on postprandial blood glucose levels when high-carbohydrate (low fat/protein) $\left(p_{\text {interaction }}=0.02\right)$ and high-fat $\left(p_{\text {interaction }}=0.03\right)$ breakfasts were consumed compared with a reference $75 \mathrm{~g}$ OGTT. Within-person sleep period time had a similar interaction (high carbohydrate: $p_{\text {interaction }}=0.001$, high fat: $\left.p_{\text {interaction }}=0.02\right)$. Within- and between-person sleep efficiency were significantly associated with lower postprandial blood glucose levels irrespective of meal type (both $p<0.03$ ). Later sleep midpoint (time deviation from midnight) was found to be significantly associated with higher postprandial glucose, in both between-person and within-person comparisons ( $p=0.035$ and $p=0.051$, respectively).

Conclusions/interpretation Poor sleep efficiency and later bedtime routines are associated with more pronounced postprandial glycaemic responses to breakfast the following morning. A person's deviation from their usual sleep pattern was also associated with poorer postprandial glycaemic control. These findings underscore sleep as a modifiable, non-pharmacological therapeutic target for the optimal regulation of human metabolic health.
\end{abstract}

Trial registration ClinicalTrials.gov NCT03479866.

Paul W. Franks

paul.franks@med.lu.se

1 Genetic \& Molecular Epidemiology Unit, Lund University Diabetes Centre, Department of Clinical Sciences, Lund University, Malmö, Sweden

2 Center for Human Sleep Science, Department of Psychology, University of California, Berkeley, CA, USA

3 Diabetes Center, Department of Medicine, Massachusetts General Hospital and Harvard Medical School, Boston, MA, USA

4 JM-USDA-Human Nutrition Research Diabetes Center on Aging at Tufts University, Boston, MA, USA

5 IMDEA-Food, Madrid, Spain

6 Clinical and Translational Epidemiology Unit, Massachusetts General Hospital and Harvard Medical School, Boston, MA, USA
7 Division of Gastroenterology, Massachusetts General Hospital and Harvard Medical School, Boston, MA, USA

8 NIHR Nottingham BRC at the Nottingham University Hospitals NHS Trust and University of Nottingham, Nottingham, UK

9 Department of Cellular, Computational and Integrative Biology, University of Trento, Trento, Italy

10 European Institute of Oncology Scientific Institute for Research, Hospitalization and Healthcare, Milan, Italy

11 Zoe Ltd, London, UK

12 Department of Nutritional Research, Kings College London, London, UK

13 Department of Twins Research, Kings College London, London, UK

14 Harvard Chan School of Public Health, Boston, MA, USA 


\section{Research in context}

\section{What is already known about this subject?}

- Sleep, diet and exercise are fundamental components of a healthy lifestyle

- The role of objectively assessed sleep in glucose homeostasis is understudied

\section{What is the key question?}

- Do night-to-night fluctuations in sleep duration, efficiency or timing impact postprandial glucose response to breakfast the following day?

\section{What are the new findings?}

- Poor sleep efficiency and later bedtime routines are associated with more pronounced postprandial glycaemic responses to breakfasts the following morning

- The study also suggests that a person's deviation from their usual sleep pattern is associated with poorer postprandial glycaemic control

\section{How might this impact on clinical practice in the foreseeable future?}

- Together, these findings underscore the importance of sleep as a modifiable, non-pharmacological therapeutic target for the optimal regulation of human metabolic health

Keywords Diet $\cdot$ Metabolic health $\cdot$ Person-centring $\cdot$ Postprandial glucose $\cdot$ Sleep

\author{
Abbreviations \\ CGM Continuous glucose monitor \\ iAUC Incremental AUC \\ PREDICT Personalized REsponses to \\ DIetary Composition Trial \\ SE $\quad$ Sleep efficiency \\ SPT Sleep period time
}

\section{Introduction}

Diet, physical activity and sleep are increasingly recognised as core modifiable components of a healthy lifestyle. Sleep disorders often coalesce with other health ailments, and in this way provide a barometer of general health [1]. Sleep has direct causal effects on many life-threatening diseases such as CVD, obesity and type 2 diabetes [2-5]. Its disturbance (such as obstructive sleep apnoea) is associated with type 2 diabetes prevalence and complications [6] and can disrupt glucose homeostasis [7]. These and other data [8-10] point to a strong link between sleep quality/duration and glucose homeostasis. However, while there have been numerous large prospective cohort studies focused on the relationship between selfreported sleep, disease and wellbeing, objective data on sleep and postprandial glucose metabolism typically emanates from small studies conducted in tightly controlled settings and in specific population subgroups such as those suffering sleep disturbances owing to pregnancy, sleep apnoea, depression, obesity or diabetes [11]. Thus, the evidence base for potential recommendations concerning the effects of sleep on glucose metabolism in generally healthy people has considerable scope for expansion.

The purpose of this study was to investigate the relationship between sleep (duration, efficiency and midpoint) and postprandial glycaemic response to breakfasts of varying macronutrient composition in healthy adults from the UK and USA. Given the established role of sleep in glucose control in people with type 2 diabetes [12], we hypothesised that shorter sleep duration and poorer quality sleep would be associated with higher $2 \mathrm{~h}$ postprandial glucose incremental AUC (iAUC). We also hypothesised that there would be an interaction between sleep and meal type, meaning that the effect of sleep on postprandial glycaemic control would be modified by the macronutrient composition of a meal. We further investigated whether within-person sleep changes (deviations from the usual sleep pattern for a given participant) predicted that individual's postprandial glycaemic control.

\section{Methods}

\section{Participants}

Participants from the UK and the USA were enrolled into the Personalized REsponses to DIetary Composition Trial 1 
(PREDICT1; ClinicalTrials.gov registration no. NCT03479866), a single-arm, multiple-test-meal challenge study conducted over 14 consecutive days. Metabolic responses to various foods differing in macronutrient and energy content were determined in relation to each participant's meal timing and sleep, as well as a range of biological characteristics [13]. The study was conducted between June 2018 and May 2019 and included 1002 generally healthy participants aged 18-65 years in the UK, as well as 100 generally healthy participants in the USA, with data being combined as there were no differences of effects for the two locations. Some of the UK participants were recruited from the TwinsUK research cohort, which includes both monozygotic twin (sharing the vast majority of their DNA sequence) and dizygotic twins (sharing roughly half of their DNA sequence) [14]. 'Healthy' was defined as being free of diagnosed diseases from the exclusion criteria. Exclusion criteria were as follows: ongoing inflammatory disease; cancer in the last 3 years (excluding skin cancer); long-term gastrointestinal disorders; taking immunosuppressants; using proton-pump inhibitors; diabetes; depression; eating disorder; and pregnancy. A full description of the PREDICT1 study protocol, including the rationale for the sample size, can be found elsewhere [13]. The current report describes secondary analyses of the PREDICT1 trial. a

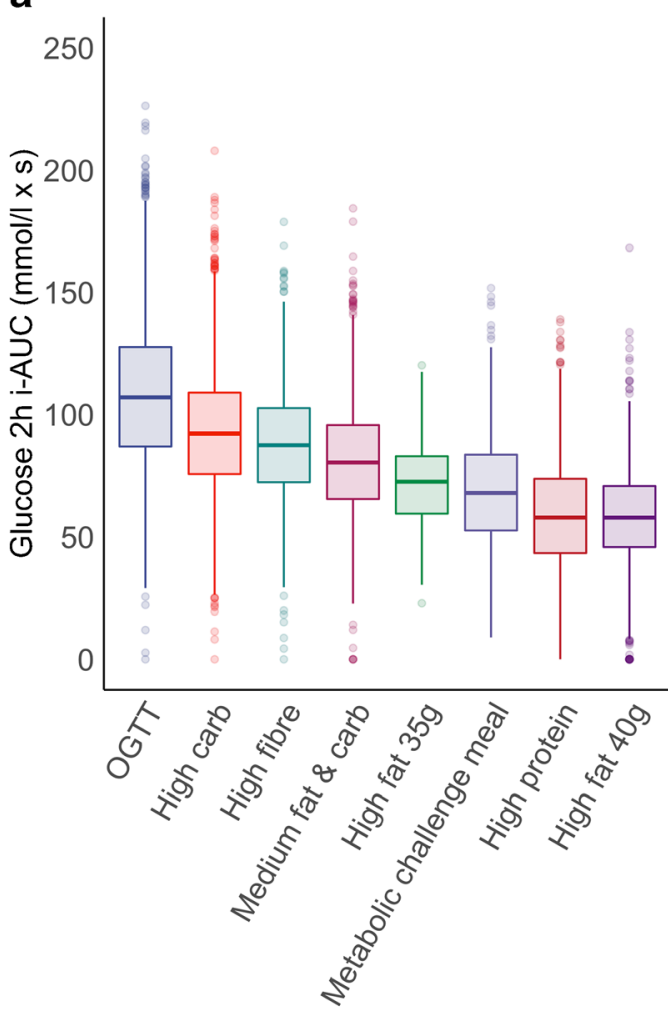

Meal type b

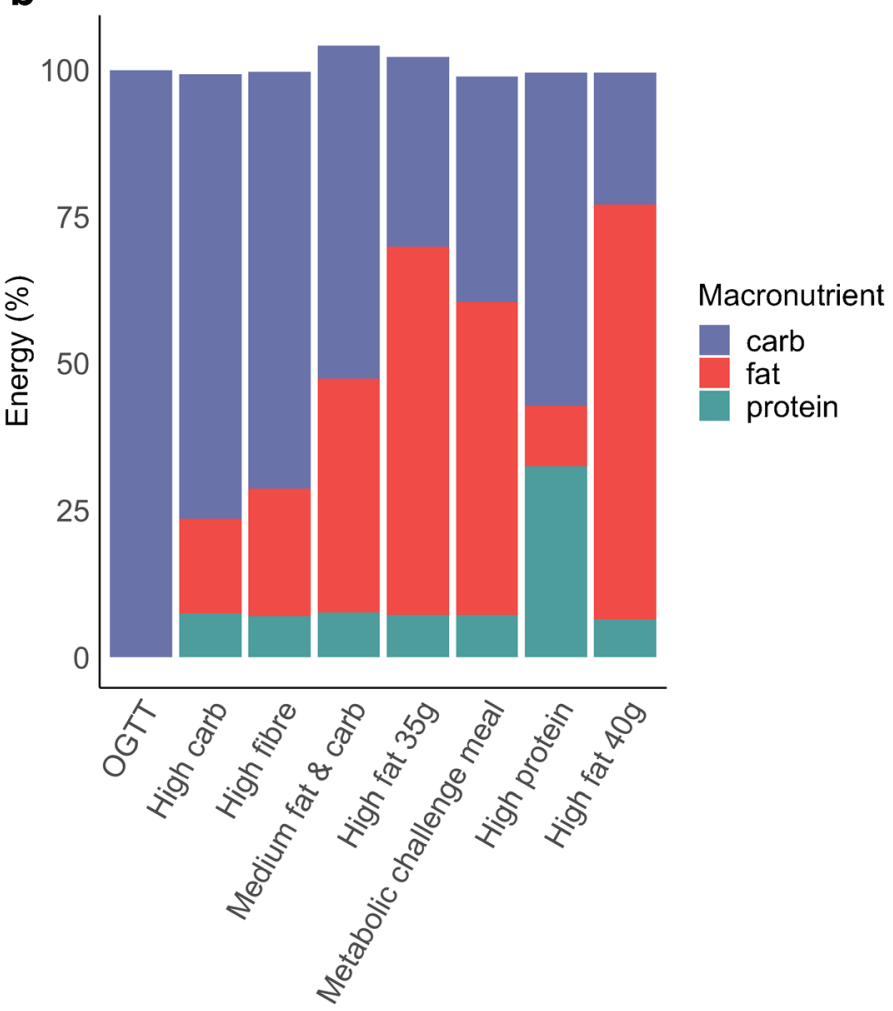

Meal type
Ethical approval for the study was obtained from the Research Ethics Committee and Integrated Research Application System (IRAS 236407) and from the Institutional Review Board (Partners Healthcare IRB 2018P002078) in the UK and USA, respectively. Written informed consent was obtained from each participant immediately prior to enrolment in the trial during the baseline clinic visit.

\section{Test meal challenges}

Participants consumed standardised test meals of different nutritional composition (carbohydrates, fat, protein and dietary fibre). The meals were consumed either for breakfast or lunch in a randomised meal order and consisted of eight different standardised meals designated as follows: (1) metabolic challenge meal; (2) medium fat and carb; (3) high fat $35 \mathrm{~g}$; (4) high carb (with low fat and protein); (5) $75 \mathrm{~g}$ OGTT, consisting of carbohydrates only; (6) high fibre; (7) high fat $40 \mathrm{~g}$; and (8) high protein. The detailed nutritional composition of the test meals can be found in Fig. 1 (see also [13]).

Participants were asked to consume only their standardised breakfasts after no less than $8 \mathrm{~h}$ of fasting and to drink only still water during the fasting period. In addition, participants 
were requested to consume all their meals within $10 \mathrm{~min}$, with the exception of the OGTT, which was to be consumed within 5 min. Participants were also requested to limit physical exercise during the $3 \mathrm{~h}$ period following the meal, as well as during the $8 \mathrm{~h}$ fasting period prior to its consumption. More detailed explanation of the test meal challenge protocol can be found elsewhere [13].

In this analysis, we included only the logged meals that complied with the protocol and passed logging accuracy assessment conducted by the study staff [13]. For example, overnight fasting was manually checked for each user, with the accuracy of meal being marked down if the time between last logged food the day before and the first on the following day was less than $8 \mathrm{~h}$. Since glucose tolerance differs across the day owing to circadian effects that are independent of sleep [15], we excluded all meals except those consumed at breakfast from the analyses, thereby reducing non-sleeprelated circadian effects on glucose regulation [15-17]. The OGTT was used as the reference meal type in the linear mixed-effects models, as the OGTT is the reference standard for assessing post-load glycaemic control in clinical settings.

\section{Sleep assessment}

Activity and sleep were monitored using a wearable device with a triaxial accelerometer (AX3, Axivity; Newcastle Upon Tyne, UK). Accelerometers were fitted by trained research staff on the non-dominant wrist and were worn by the participants for the duration of the entire study.

Raw accelerometer data were analysed using GGIR, a multi-day raw accelerometer data analysis package in $\mathrm{R}[18$, 19]. The raw accelerometer data from a wrist actigraphy units were converted into a single activity time series (BFEN) aggregated into epochs of $30 \mathrm{~s}$. Sleep/wake detection was performed using the validated angle-z method implemented in GGIR [19]. Based on prior evidence linking sleep with metabolic system regulation [12], primary a priori target variables were as follows: (1) sleep duration or total sleep period time (SPT); (2) sleep efficiency (SE), where SE represents the ratio of time asleep to the total SPT; and (3) sleep midpoint, or the middle time point between bedtime and waking up (expressed in hours as a deviation from midnight). Sleep predictors were further decomposed into within-person effects (as opposed to between-person effects) using person-mean centring [20]. In the context of this study, such person-mean centring translated into the person's 2 week average and his/ her deviation from that average on a particular day.

A set of filtering conditions consistent with the practices of typical sleep analysis was applied to remove entries with invalid sleep data [21]. Nights with $<2 \mathrm{~h}$ or $>15 \mathrm{~h}$ of sleep or with SE $<20 \%$ were excluded from the analysis, as they likely resulted from artefacts or poor-quality data (355 nights [2.5\%]). Nights with more than $10 \%$ data classified as invalid were also excluded (399 nights [2.8\%]). In addition, nights with a sleep onset during 08:00-17:00 h or a sleep offset after 00:00 h were excluded (26 nights [0.2\%]). Finally, data from participants with less than 7 days of data and/or a percentage of invalid nights $>35 \%(n=89)$ were removed. We also removed data from participants who travelled in different time zones during the study, owing to disruption of their regular sleep patterns.

\section{Glucose assessment}

Postprandial blood glucose was computed using a continuous glucose monitoring (CGM) wearable device (Freestyle Libre Pro; Abbott, Abbott Park, IL, USA), which measures interstitial glucose every $15 \mathrm{~min}$. Monitors were fitted by trained research staff to the participant's upper non-dominant arm and were worn for the entire study duration. Owing to the CGM's calibration requirements, CGM data collected $12 \mathrm{~h}$ after fitting the device to a participant was used for analysis [13].

Incremental area under the blood glucose curve has been shown to accurately describe glycaemic responses to foods [22]. Accordingly, the primary outcome for this analysis was $2 \mathrm{~h}$ iAUC for postprandial glucose with interpolated baseline (glucose $\mathrm{i}_{\mathrm{iAUC}}$ $2 \mathrm{~h}$ ). The baseline value was interpolated at the meal start time based on the nearest CGM readings immediately before and after that time. The distribution of glucose $\mathrm{i}_{\mathrm{AUC} C-2 \mathrm{~h}}$ was right skewed. Thus, these data were transformed using a square-root transformation, which yielded better normalisation than conventional logarithmic transformations (electronic supplementary material [ESM] Fig. 1).

\section{Statistical analysis}

Data analysis was performed using $\mathrm{R}$ version 3.6.1 [23]. To account for the individual uniqueness/variation of postprandial glycaemic responses (inter-individual variability) and the covariance structure resulting from repeated measurements (which would violate the independence of observations assumption necessary for linear regression), data were modelled using a linear mixed-effects model approach. We analysed data using lme4 package with lme4's default REML (restricted or 'residual' maximum likelihood) criterion to estimate variance components [24]. The lmerTest package was used to calculate $p$ values [25]; $p<0.05$ was considered statistically significant.

All models were initially constructed with only the marginal terms for sleep and meal; the multiplicative interaction term for sleep $\times$ meal was added to the subsequent models. Similarly, all between-person models were subsequently rerun using the within-person approach. The outcome was glucose $_{\mathrm{iAUC0}-2 \mathrm{~h}}$. Potential confounders were also taken into consideration. The models consisted of nine fixed effects: (1) different between-person and within-person sleep 
Table 1 Participant characteristics

\begin{tabular}{llll}
\hline Characteristic & $\begin{array}{l}\text { PREDICT1 UK } \\
(n=869)\end{array}$ & $\begin{array}{l}\text { PREDICT1 USA } \\
(n=84)\end{array}$ & $\begin{array}{l}\text { Overall } \\
(n=953)\end{array}$ \\
\hline Sex, $n(\%)$ & $636(73.2)$ & $55(65.5)$ & $691(72.5)$ \\
$\quad$ Female & $233(26.8)$ & $29(34.5)$ & $262(27.5)$ \\
Male & & & \\
Age, years & $46.2 \pm 11.9$ & $41.8 \pm 12.8$ & $45.8 \pm 12.0$ \\
Zygosity, $n(\%)$ & & & \\
NT & $303(34.9)$ & $84(100)$ & $387(40.6)$ \\
DZ & $151(17.4)$ & $0(0)$ & $151(15.8)$ \\
MZ & $415(47.8)$ & $0(0)$ & $415(43.5)$ \\
BMI, kg/m ${ }^{2}$ & $25.7 \pm 5.01$ & $25.7 \pm 4.26$ & $25.7 \pm 4.95$ \\
SE, \% ${ }^{\mathrm{a}}$ & $0.89 \pm 0.057$ & $0.89 \pm 0.062$ & $0.89 \pm 0.058$ \\
SPT, $\mathrm{h}^{\mathrm{a}}$ & $7.69(6.87,8.48)$ & $7.64(6.66,8.61)$ & $7.68(6.86,8.50)$ \\
Sleep midpoint as a deviation from midnight, $\mathrm{h}^{\mathrm{a}}$ & $3.25 \pm 1.15$ & $2.87 \pm 1.30$ & $3.21 \pm 1.17$ \\
Glucose 2 h iAUC, mmol/1 $\times \mathrm{s}^{\mathrm{a}}$ & $83.0 \pm 31.3$ & $81.5 \pm 32.6$ & $82.8 \pm 31.4$ \\
Meal type, $n(\%)^{\mathrm{a}}$ & & & \\
OGTT & $1507(19.8)$ & $136(17.2)$ & $1643(19.6)$ \\
High carb & $1475(19.4)$ & $140(17.7)$ & $1615(19.2)$ \\
High fat $35 \mathrm{~g}$ & $215(2.8)$ & $0(0)$ & $215(2.6)$ \\
High fat $40 \mathrm{~g}$ & $1105(14.5)$ & $144(18.2)$ & $1249(14.9)$ \\
High fibre & $683(9.0)$ & $76(9.6)$ & $759(9.0)$ \\
High protein & $844(11.1)$ & $75(9.5)$ & $919(10.9)$ \\
MCM & $319(4.2)$ & $71(9.0)$ & $390(4.6)$ \\
Medium fat and carb & $1457(19.2)$ & $148(18.7)$ & $1605(19.1)$ \\
\hline
\end{tabular}

Values are mean $\pm \mathrm{SD}$, median $(\mathrm{Q} 1, \mathrm{Q} 3)$

${ }^{\mathrm{a}}$ meals $n=7605$ for PREDICT1 UK, $n=790$ for PREDICT1 USA and $n=8395$ overall)

DZ, dizygotic twin; MCM, metabolic challenge meal; MZ, monozygotic twin; NT, not a twin variables (SE, SPT, sleep midpoint); (2) standardised meal type; (3) sex; (4) age; (5) BMI; (6) zygosity (not a twin, monozygotic, dizygotic); (7) weekend; and (8) season; and (9) when applicable, sleep variable and standardised meal type interaction term to assess the combined effect of sleep variable and meal type on postprandial glucose metabolism. For random effects terms, we used participant ID (randomly generated study identification [ID] number) and family ID to allow for person-specific linear regressions, where participants have their own intercepts for the association of interest. Participant ID was nested within family ID to account for the data's nested/hierarchical structure and the fact that measures coming from twins belonging to the same family might be more similar to each other owing to genetic and/or environmental factors. The models had random intercepts but no random slopes. Visual inspection of diagnostic plots did not reveal any strong deviations from homoscedasticity or normality, and all models were checked for multicollinearity. The main hypotheses tested were as follows: (1) whether the relationship between sleep (quality, timing or duration) and blood glucose is statistically different from the null hypothesis of no effect; and (2) whether the relationship between sleep and postprandial glycaemic response differs in magnitude conditional on the type of breakfast consumed. ESM Methods provides $\mathrm{R}$ codes for each of the main hypothesis tests. Owing to the hypothesis-driven nature of the analyses reported here, we considered a nominal $p$ value threshold of 0.05 to be of statistical significance.

\section{Results}

\section{Participant characteristics}

Data from 953 participants were used to analyse 8395 sleep and postprandial responses (Fig. 1, Table 1). Consort diagrams for the PREDICT1 study can be found elsewhere [26].

To provide insights into the effects of sleep on postprandial glycaemic control at both within-individual and group level, we present these two sets of results separately. All models were adjusted for age, sex, BMI, meal type, zygosity, weekend and season. We found no significant interactions between postprandial glucose and sex, meaning that there was no evidence to believe the results are different for the two sexes. 


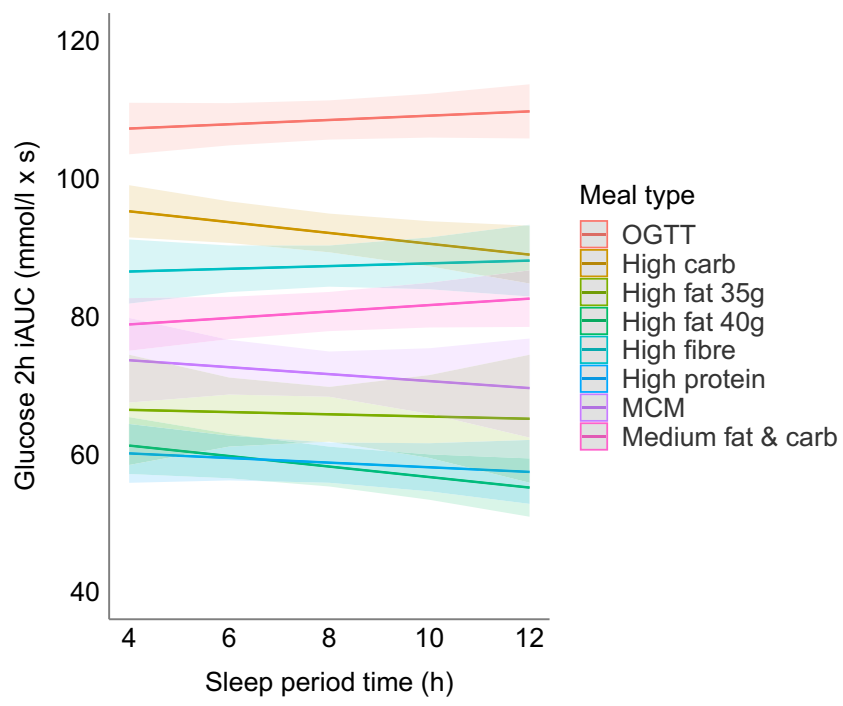

Fig. 2 Meal type and SPT interaction effects on glucose $2 \mathrm{~h}$ iAUC. The plot fits the model using the marginal effects of the interaction terms with the standard errors. MCM, metabolic challenge meal

\section{SPT}

Between-participant effects We found no statistically significant association between SPT and glucose $\mathrm{iAUC0}_{\mathrm{AU}}$ (ESM Table 1). However, we found a statistically significant interaction between SPT and meal type, with SPT having a significantly negative association with glucose $\mathrm{iAUCO}_{\mathrm{AU}-2 \mathrm{~h}}$ following high-carbohydrate and high-fat breakfasts $\left(\beta_{\mathrm{SPT}} \times\right.$ high carb $=-1.10, p_{\mathrm{SPT}} \times$ high carb $=0.021 ; \beta_{\mathrm{SPT}} \times$ high fat $40 \mathrm{~g}=$ $-1.07, p_{\mathrm{SPT}} \times$ high fat $\left.40 \mathrm{~g}=0.031\right)($ Fig. 2 and ESM Table 1$)$.

Within-participant effects The person-centred model revealed an effect similar to that of the between-person model, suggesting that an SPT that exceeded a person's average SPT is associated with lower postprandial response following a highcarbohydrate or high-fat breakfast $\left(\beta_{\mathrm{SPT} \text {-person }} \times\right.$ high carb $=$ $-1.84, p_{\mathrm{SPT} \text {-person }} \times$ high carb $=0.001 ; \beta_{\mathrm{SPT} \text {-person }} \times$ high fat $\left.40 \mathrm{~g}=-1.36, p_{\text {SPT-person } \times \text { high fat } 40 \mathrm{~g}}=0.019\right)($ ESM Table 2$)$.

\section{SE}

Between-participant effects A larger between-person SE was significantly associated with lower glucose $\mathrm{iAUC0}_{\mathrm{AU}-2 \mathrm{~h}}\left(\beta_{\mathrm{SE}}=\right.$ -10.48 [95\% CI $\left.-19.85,-1.11], p_{\mathrm{SE}}=0.028\right)(\mathrm{ESM}$ Table 3). In a model assessing the interaction of SE and meal type, higher SE was still significantly associated with lower glucose $_{\text {iAUC0-2h }}\left(\beta_{\mathrm{SE}}=-19.18[95 \% \mathrm{CI}-36.46,-1.91], p_{\mathrm{SE}}\right.$ $=0.030$ ), but none of the interaction terms was statistically significant (ESM Table 3). This association remained similar after additional adjustment for SPT ( $p=0.016)$.

Table 2 Between-person sleep midpoint and postprandial blood glucose concentrations

\begin{tabular}{|c|c|c|c|c|c|c|}
\hline \multirow[t]{2}{*}{ Predictor } & \multicolumn{3}{|c|}{ Model without interaction } & \multicolumn{3}{|c|}{ Model with interaction } \\
\hline & Estimate & $95 \% \mathrm{CI}$ & $p$ value & Estimate & $95 \% \mathrm{CI}$ & $p$ value \\
\hline (Intercept) & 81.13 & $72.61,89.65$ & $<0.001$ & 80.70 & $71.90,89.51$ & $<0.001$ \\
\hline Sleep midpoint ${ }^{\mathrm{a}}$ & 0.52 & $0.04,1.01$ & 0.035 & 0.69 & $-0.17,1.55$ & 0.116 \\
\hline Meal (high carb) & -15.92 & $-17.23,-14.60$ & $<0.001$ & -14.83 & $-18.74,-10.93$ & $<0.001$ \\
\hline Meal (high fat $35 \mathrm{~g}$ ) & -42.42 & $-45.38,-39.46$ & $<0.001$ & -41.66 & $-50.39,-32.93$ & $<0.001$ \\
\hline Meal (high fat $40 \mathrm{~g}$ ) & -49.98 & $-51.39,-48.58$ & $<0.001$ & -48.17 & $-52.31,-44.03$ & $<0.001$ \\
\hline Meal (high fibre) & -21.20 & $-22.82,-19.57$ & $<0.001$ & -17.62 & $-22.41,-12.83$ & $<0.001$ \\
\hline Meal (high protein) & -49.56 & $-51.11,-48.01$ & $<0.001$ & -51.21 & $-55.71,-46.71$ & $<0.001$ \\
\hline Meal (MCM at home) & -36.55 & $-38.67,-34.43$ & $<0.001$ & -33.10 & $-39.73,-26.46$ & $<0.001$ \\
\hline Meal (medium fat and carb) & -27.90 & $-29.19,-26.60$ & $<0.001$ & -29.06 & $-32.94,-25.18$ & $<0.001$ \\
\hline Sleep midpoint $\times$ Meal (high carb) & & & & -0.33 & $-1.46,0.80$ & 0.565 \\
\hline Sleep midpoint $\times$ Meal (high fat $35 \mathrm{~g}$ ) & & & & -0.23 & $-2.77,2.31$ & 0.860 \\
\hline Sleep midpoint $\times$ Meal (high fat $40 \mathrm{~g}$ ) & & & & -0.58 & $-1.82,0.66$ & 0.358 \\
\hline Sleep midpoint $\times$ Meal (high fibre) & & & & -1.09 & $-2.46,0.29$ & 0.121 \\
\hline Sleep midpoint $\times$ Meal (high protein) & & & & 0.49 & $-0.79,1.77$ & 0.455 \\
\hline Sleep midpoint $\times$ Meal (MCM at home) & & & & -1.13 & $-3.19,0.92$ & 0.280 \\
\hline Sleep midpoint $\times$ & & & & 0.37 & $-0.77,1.51$ & 0.525 \\
\hline
\end{tabular}

Random effects terms for both models: intraclass correlation coefficient, $0.45 ; N=953_{\text {username }} ; N=765_{\text {family id }} ; n$ observations $8395 ;$ marginal $R^{2} /$ conditional $R^{2}=0.363 / 0.652$

${ }^{\text {a }}$ Sleep midpoint is expressed in hours as a deviation from midnight

MCM, Metabolic challenge meal 
Within-participant effects A greater within-person SE was significantly associated with lower glucose $\mathrm{iAUC0}_{\mathrm{A}-2 \mathrm{~h}}\left(\beta_{\mathrm{SE}}=\right.$ $\left.-11.93\left[95 \% \mathrm{CI}_{\mathrm{SE}}-21.83,-2.04\right], p_{\mathrm{SE}}=0.018\right)$, meaning that achieving better than one's average SE was associated with better postprandial glycaemic control the following day (ESM Table 4). In the model with SE and meal type interaction term, higher within-person SE was still significantly associated with lower glucose $\mathrm{iAUC0}_{\mathrm{A}-2 \mathrm{~h}}\left(\beta_{\mathrm{SE}}=-29.75\left[95 \% \mathrm{CI}_{\mathrm{SE}}\right.\right.$ $\left.-52.29,-7.21], p_{\mathrm{SE}}=0.010\right)($ ESM Table 4$)$.

\section{Sleep midpoint}

Between-participant and within-participant effects A later between-person sleep midpoint (expressed in hours as a deviation from midnight) was significantly associated with higher glucose iAUC $\left(\beta_{\text {midpoint }}=0.52\right.$ [95\% $\left.\mathrm{CI}_{\text {midpoint }} 0.04,1.01\right]$, $\left.p_{\text {midpoint }}=0.035\right)($ Table 2$)$. This effect was largely driven by sleep onset (going to bed later) rather than sleep offset (waking up later) (ESM Tables 5-7). The effect ceased to be significant in a model incorporating an interaction term between sleep midpoint and meal type (Table 2). In the within-person effects model, having a later sleep midpoint than one's average had a similar coefficient $\left(\beta_{\text {midpoint }}=\right.$ $\left.0.51\left[95 \% \mathrm{CI}_{\text {midpoint }} 0.00,1.02\right], p_{\text {midpoint }}=0.051\right)$.

\section{Discussion}

Here, we describe for the first time how sleep duration, quality and midpoint associate with postprandial glucose metabolism in healthy individuals. While sleep is generally recognised as one of the pillars of good health, the data reported here suggest that one-size-fits-all sleep recommendations are suboptimal, particularly in the context of postprandial glycaemic control, a key component of diabetes prevention.

By analysing both between-person and within-person effects, this study provides unique and powerful insights into both population-level and person-level effects of sleep on metabolic health. Notably, our data suggest that sleep duration, efficiency and midpoint are important determinants of postprandial glycaemic control at a population level, while illustrating that to optimise sleep recommendations it is likely necessary to tailor these to the individual.

Diet, sleep and health are interrelated. Several studies have investigated the relationship between sleep duration and glucose metabolism in pregnant women and reported a positive association between reduced sleep duration and impaired glucose metabolism [4, 27]. However, we are not aware of any other studies to date that investigate the relationship between objectively assessed sleep characteristics and postprandial glucose metabolism in generally healthy adults. The findings from this intervention study, with repeated test meal challenges, combined with objective assessments of sleep and blood glucose from a large population, complement a relatively small body of knowledge around a topic that is likely to be of high relevance for diabetes prevention [26]. Importantly, many earlier studies were undertaken in sleep laboratories with small sample size [11]. While the controlled environment of such studies is necessary to understand specific aspects of sleep and metabolism, the real-world, community-dwelling setting of the current study provides novel insights into how habitual sleep affects metabolic health.

The main analyses in this study focused on interactions of sleep and meal type and selected the OGTT as the 'breakfast' against which all other breakfast meals were compared. This is primarily because the OGTT is the standard clinical test used to assess glucose tolerance. While this is not a realistic breakfast meal, there is a growing trend, particularly among younger people [28], to consume energy drinks as a pick-me-up the morning after a poor night's sleep, with the sugar content of a $75 \mathrm{~g}$ OGTT equating to roughly two to three servings of standard energy drinks.

\section{SPT}

With SPT being a marker of sleep duration, the lack of a significant marginal effect in the model without interactions indicates that sleep duration is not a major determinant of glucose metabolism. While this finding does not support some prior studies that have demonstrated a potential link between decreased sleep duration and insulin resistance, it is consistent with findings from a randomised controlled trial of 42 normalweight adult short sleepers [11]. This may be because the effect of sleep duration in glycaemic control may be non-linear, with sleep affecting glucose metabolism only once sleep duration dips below a specific bound [11]. Moreover, sleep duration for the vast majority of PREDICT1 participants fell within the recommended range, as indicated by the mean $6.87 \mathrm{~h}$ of sleep within the lowest quartile of the SPT distribution (Table 1). Accordingly, this study may have been underpowered to detect an association between sleep duration and postprandial glycaemic control.

We found a significant statistical interaction between SPT and meal type, with high-carbohydrate meals and high-fat meals resulting in significantly lower glucose iAUCs compared with the OGTT reference, which contained only sugar. Although this study did not include pregnant women, the interaction effect between carbohydrate-rich meals and SPT is consistent with the results reported in a prior study in which reduced sleep was associated with impaired carbohydrate metabolism in pregnant women [29]. Thus, we conclude that the SPT has a similar impact on postprandial carbohydrate metabolism in men and pregnant and non-pregnant women. Additionally, the significant interaction between SPT and high-fat meals is supported by the finding that sleep disruption in fat-fed mice negatively affected glucose metabolism, with metabolism improving after recovery 
sleep [30]. As for the within-person SPT model, our findings suggest that both a longer SPT in general, as well as having a longer SPT than one is used to, are associated with improved postprandial glycaemic control the following morning. The presence of a significant finding with the SPT $\times$ high-carbohydrate meal interaction term in the person-centred model suggests that getting more sleep than usual might be more important for postprandial glycaemic control than the absolute amount of sleep achieved. This insight offers a potential avenue for personalised (within-person) sleep interventions.

\section{SE}

Better SE, between-person as well as within-person, was significantly associated with lower glucose iAUC, meaning that better SE, which is a proxy of sleep quality, was associated with better glucose management following breakfast. However, the absence of SE $\times$ meal interactions suggests that SE may be beneficial for postprandial glucose response irrespective of meal composition. Although there is not much research on SE and glucose metabolism in healthy adults, our findings concur with a recent metaanalysis in which poor sleep quality was associated with poor glycaemic control in individuals with type 2 diabetes [12]. Moreover, since SE can be viewed as a proxy for sleep quality, and because we found a significant association between SE and glucose regulation, but not between SPT and glucose regulation, our findings suggest that sleep quality is more important than sleep duration with respect to glycaemic control. However, sleep apnoea is known to affect SE, and sleep apnoea was not measured in the PREDICT1 study.

\section{Sleep midpoint}

The presence of significant effects in both between-person and within-person sleep midpoint models adjusted for sleep duration suggests a novel finding that later sleep midpoint, such as that caused by going to bed later, is associated with impaired postprandial glucose response to breakfast the following morning. This concurs with the proposition that human metabolic health is determined to a considerable extent by chronobiology [31]. Alternatively, later sleep midpoint may reflect alteration of sleep stages caused by going to bed later. Thus, the significance of later sleep midpoint may also be indicative of the role of specific sleep stages, such as slow-wave sleep, on glucose metabolism, supporting the view that treating slow-wave sleep disorders may help improve glycaemic control [32].

The $2 \mathrm{~h}$ glucose iAUC response to an OGTT breakfast is roughly twice that following a high-fat breakfast, indicating that a high-fat breakfast might help to mitigate the detrimental effects of poor sleep on postprandial glycaemia. Although comparing areas may have its drawbacks, for those whose sleep is often compromised, these effects may be cumulative.
Thus, over time, there may be a meaningful clinical impact on glycaemic health. Nevertheless, because of the short duration of the current study, we are not able to assess this hypothesis.

Much of the research linking poor sleep with altered glucose metabolism is based upon observational studies, meaning that the pathophysiological mechanisms behind the associations reported here are not well understood [33]. However, poor sleep quality (measured by sleep fragmentation in healthy volunteers) appears to alter glucose responses through shifting sympathovagal balance and morning cortisol levels, which could in turn lead to decreased insulin sensitivity, increased hepatic glucogenesis and decreased insulin secretion [33]. In addition to cortisol levels, growth hormone, the secretion of which is sleep-dependent and which is essential for metabolic regulation, could also be at play [34-36]. Moreover, the glucose intolerance observed elsewhere in sleep-deprived individuals may derive from dysregulation of sympathetic and parasympathetic control of pancreatic function [37].

\section{Strengths and limitations}

This study significantly extends our understanding of the interplay between sleep and metabolic health. First, the fairly limited literature on sleep and postprandial blood glucose regulation is dominated by small studies that focus on populations with comorbid conditions (e.g. diabetes and obstructive sleep apnoea). In the few larger published studies, sleep has typically been assessed through self-report, which may be prone to bias. Moreover, most studies are cross-sectional and based in highly controlled environments. By contrast, our study is set within a large prospective cohort of generally healthy individuals, in whom high-resolution objectively assessed time-series sleep and glucose data were obtained. These design features made it possible to look at both intraand inter-individual variation during the analyses, have generalisable results and shed light on cause and effect. The risk of non-compliance due to a non-clinical setting was addressed by high levels of staff support and all data points were checked for compliance and validity. We also examined the effects of within-person sleep measures, thus broadening the scope of previous studies that up to now only included between-person differences. In addition, instead of relying on fasting blood assays, the study focused on postprandial glucose, which is more relevant to everyday life scenarios because most people find themselves most often in a postprandial state during waking hours [26].

Notwithstanding the strengths of the study, it is limited in that no screening was performed for sleep disorders (e.g. sleep apnoea and insomnia), meaning that we could not control for disorders that have been shown to be associated with impaired glucose tolerance [38, 39]. In addition, while actigraphy overcomes many limitations of self-report measures, it is not as 
accurate as polysomnography in estimating sleep duration and efficiency and does not offer insight into individual sleep stages. The distribution of meal types was imbalanced, with high-fat and high-carbohydrate standardised meals having the highest number of entries. Thus, it is possible that analyses focused on the non-high-carbohydrate and non-high-fat meals may have lacked statistical power. An additional limitation is that owing to the free-living nature of the trial, physical activity levels varied within and between individuals, which may have interacted with sleep and meal type to affect blood glucose concentrations, a hypothesis that our study was not powered to examine.

Future studies assessing the impact of sleep stages on postprandial blood glucose levels are likely to extend the findings of the current analysis, as would exploration of these effects in individuals who are sleep-deprived owing to shift work or endogenous sleep disorders such as sleep apnoea.

\section{Conclusion}

Overall, this study suggests that sleep duration, quality and midpoint are important modifiable lifestyle features for improving postprandial glucose metabolism in healthy adults. As a consequence, this study's findings may inform lifestyle strategies to improve postprandial blood glucose levels, focusing on earlier bedtime routines and maximising high-quality uninterrupted sleep. A combination of both generalised and more personalised sleep guidelines is likely required to ensure optimal metabolic health per se and maximise the effectiveness of guidelines for diabetes prevention.

Supplementary Information The online version contains peer-reviewed but unedited supplementary material available at https://doi.org/10.1007/ s00125-021-05608-y.

Acknowledgements We thank the participants of the PREDICT1 study. We also thank the staff of Zoe Ltd., the Department of Twin Research and Genetic Epidemiology, and Massachusetts General Hospital and the Translational and Clinical Research Center for their tireless work in contributing to the running of the study and data collection. We thank Abbott for their support with using their CGMs.

Data availability The data from the baseline clinic visit of PREDICT1 belong to the department of Twin Research at King's College London. Information about the data access application process can be found at: https://twinsuk.ac.uk/resources-for-researchers/access-our-data/.

The data from the at-home phase of the trial, which belong to Zoe Ltd. and were used under license, are not publicly available. However, the data can be made available granted a reasonable request and a permission from Zoe Ltd.

Funding Open access funding provided by Lund University. This work was supported by Zoe Ltd. TwinsUK is funded by the Wellcome Trust, Medical Research Council, European Union, Chronic Disease Research Foundation (CDRF), Zoe Global Ltd. and the National Institute for Health Research (NIHR)-funded BioResource, Clinical Research Facility and Biomedical Research Centre based at Guy's and St
Thomas' NHS Foundation Trust in partnership with King's College London.

This study was supported by Zoe Ltd., Wellcome Trust and NIHR. The PREDICT trial was conducted as a collaboration between academic research scientists and Zoe Ltd., the latter of which is a health data science start-up that spun out of Kings College London. As such, some of the funding for the PREDICT trial was provided by Zoe Ltd., who also contributed to the design and implementation of the study protocol, as well as the processing, analysis and interpretation of data. PWF holds a European Research Council consolidator award (CoG-2015_681742_ NASCENT).

Authors' relationships and activities TDS, SEB, LMD and PWF are consultants to Zoe Ltd. ('Zoe'). JW is an employee of Zoe. MPW serves as a consultant for and has equity interest in Oura, Bryte, Shuni and StimScience. All other authors declare that there are no relationships or activities that might bias, or be perceived to bias, their work.

Contribution statement TDS, SEB, AMV, JW and PWF conceptualised and designed the PREDICT trial. NT, RV, JFT and PWF contributed to data analysis. NT, RV, JFT, PWF, LMD, SEB, MPW, TDS, JW and JMO contributed to analysis interpretation and the discussion. NT and PWF wrote the manuscript. All authors reviewed/edited the manuscript and all approved the final version. The guarantor, PWF, accepts full responsibility for the work and/or conduct of the study, had access to the data, and controlled the decision to publish.

Open Access This article is licensed under a Creative Commons Attribution 4.0 International License, which permits use, sharing, adaptation, distribution and reproduction in any medium or format, as long as you give appropriate credit to the original author(s) and the source, provide a link to the Creative Commons licence, and indicate if changes were made. The images or other third party material in this article are included in the article's Creative Commons licence, unless indicated otherwise in a credit line to the material. If material is not included in the article's Creative Commons licence and your intended use is not permitted by statutory regulation or exceeds the permitted use, you will need to obtain permission directly from the copyright holder. To view a copy of this licence, visit http://creativecommons.org/licenses/by/4.0/.

\section{References}

1. Zee PC, Turek FW (2006) Sleep and health: everywhere and in both directions. Arch Intern Med 166(16):1686-1688. https://doi.org/10. 1001/archinte.166.16.1686

2. GBD 2017 Disease and Injury Incidence and Prevalence Collaborators (2019) Health effects of dietary risks in 195 countries, 1990-2017: a systematic analysis for the global burden of disease study 2017. Lancet 393(10184):1958-1972. https://doi. org/10.1016/S0140-6736(19)30041-8

3. Chattu VK, Manzar MD, Kumari S, Burman D, Spence DW, Pandi-Perumal SR (2018) The global problem of insufficient sleep and its serious public health implications. Healthcare. 7(1):1

4. Facco FL, Grobman WA, Kramer J, Ho KH, Zee PC (2010) Selfreported short sleep duration and frequent snoring in pregnancy: impact on glucose metabolism. Am J Obstet Gynecol 203(2):142 e1-5

5. Blaak EE, Antoine JM, Benton D et al (2012) Impact of postprandial glycaemia on health and prevention of disease. Obes Rev 13(10):923-984. https://doi.org/10.1111/j.1467-789X.2012. 01011.x 
6. Reutrakul S, Mokhlesi B (2017) Obstructive sleep apnea and diabetes: a state of the art review. Chest. 152(5):1070-1086. https://doi. org/10.1016/j.chest.2017.05.009

7. Mokhlesi B, Tjaden AH, Temple KA et al (2021) Obstructive sleep apnea, glucose tolerance, and $\beta$-cell function in adults with prediabetes or untreated type 2 diabetes in the restoring insulin secretion (RISE) study. Diabetes Care 44(4):993-1001. https://doi.org/10. 2337/dc20-2127

8. Kim D, Hoyos CM, Mokhlesi B, Pamidi S, Jonathan C (2020) Editorial: metabolic health in normal and abnormal sleep. Front Endocrinol 11:131

9. Koren D, Levitt Katz LE et al (2011) Sleep architecture and glucose and insulin homeostasis in obese adolescents. Diabetes Care 34(11):2442-2447. https://doi.org/10.2337/dc11-1093

10. Toyoura M, Miike T, Tajima S, Matsuzawa S, Konishi Y (2020) Inadequate sleep as a contributor to impaired glucose tolerance: a cross-sectional study in children, adolescents, and young adults with circadian rhythm sleep-wake disorder. Pediatr Diabetes 21(4):557-564. https://doi.org/10.1111/pedi.13003

11. Kothari V, Cardona Z, Chirakalwasan N, Anothaisintawee T, Reutrakul S (2021) Sleep interventions and glucose metabolism: systematic review and meta-analysis. Sleep Med 78:24-35. https://doi.org/10.1016/j.sleep.2020.11.035

12. Lee SWH, Ng KY, Chin WK (2017) The impact of sleep amount and sleep quality on glycemic control in type 2 diabetes: a systematic review and meta-analysis. Sleep Med Rev 31:91-101. https:// doi.org/10.1016/j.smrv.2016.02.001

13. Berry S, Drew D, Linenberg I et al (2020) Personalised REsponses to DIetary composition trial (PREDICT): an intervention study to determine inter-individual differences in postprandial response to foods. Protoc Exch. https://doi.org/10.21203/rs.2.20798/v1

14. Department of Twin Research \& Genetic Epidemiology - King's College London UK. Twins UK. Available from: https://twinsuk. ac.uk/. Accessed Mar 312021

15. Morris CJ, Yang JN, Garcia JI et al (2015) Endogenous circadian system and circadian misalignment impact glucose tolerance via separate mechanisms in humans. Proc Natl Acad Sci U S A 112(17):E2225-E2234. https://doi.org/10.1073/pnas.1418955112

16. Saad A, Dalla Man C, Nandy DK et al (2012) Diurnal pattern to insulin secretion and insulin action in healthy individuals. Diabetes. 61(11):2691-2700. https://doi.org/10.2337/db11-1478

17. Takahashi M, Ozaki M, Kang M-I et al (2018) Effects of meal timing on postprandial glucose metabolism and blood metabolites in healthy adults. Nutrients. 10(11):1763. https://doi.org/10.3390/ nu10111763

18. Migueles JH, Rowlands AV, Huber F, Sabia S, van Hees VT (2019) GGIR: a research community-driven open source R package for generating physical activity and sleep outcomes from multiday raw accelerometer data. J Meas Phys Behav 2(3):188-196

19. van Hees VT, Sabia S, Jones SE et al (2018) Estimating sleep parameters using an accelerometer without sleep diary. Sci Rep 8(1): 12975

20. Lijuan W, Maxwell SE (2015) On disaggregating between-person and within-person effects with longitudinal data using multilevel models. Psychol Methods 20(1):63-83

21. Jones SE, van Hees VT, Mazzotti DR et al (2019) Genetic studies of accelerometer-based sleep measures yield new insights into human sleep behaviour. Nat Commun 10(1):1-12

22. Le Floch JP, Escuyer P, Baudin E, Baudon D, Perlemuter L (1990) Blood glucose AUC. Methodological aspects. Diabetes Care 13(2): 172-175. https://doi.org/10.2337/diacare.13.2.172
23. R Core Team (2019) R: A language and environment for statistical computing. R Foundation for Statistical Computing, Vienna, Austria. URL https://www.R-project.org/

24. Bates D, Mächler M, Bolker B, Walker S (2015) Fitting linear mixed-effects models using lme4. J Stat Softw 67(1):1-48

25. Kuznetsova A, Brockhoff PB, Christensen RHB (2017) \{lmerTest\} package: tests in linear mixed effects models. J Stat Softw 82(13): $1-26$

26. Berry SE, Valdes AM, Drew DA et al (2020) Human postprandial responses to food and potential for precision nutrition. Nat Med 26(6):964-973. https://doi.org/10.1038/s41591-020-0934-0

27. Twedt R, Bradley M, Deiseroth D, Althouse A, Facco F (2015) Sleep duration and blood glucose control in women with gestational diabetes mellitus. Obstet Gynecol 126(2):326-331. https://doi.org/ 10.1097/AOG.0000000000000959

28. Luneke AC, Glassman TJ, Dake JA, Blavos AA, Thompson AJ, Kruse-Diehr AJ (2020) Energy drink expectancies among college students. J Am Coll Health 1-9. https://doi.org/10.1080/07448481. 2020.1790569

29. O'Keeffe M, St-Onge MP (2013) Sleep duration and disorders in pregnancy: implications for glucose metabolism and pregnancy outcomes. Int J Obes (2005) 37(6):765-770. https://doi.org/10. 1038/ijo.2012.142

30. Ho JM, Barf RP, Opp MR (2016) Effects of sleep disruption and high fat intake on glucose metabolism in mice. Psychoneuroendocrinology. 68:47-56. https://doi.org/10.1016/j.psyneuen.2016.02.024

31. Stenvers DJ, Scheer FAJL, Schrauwen P, la Fleur SE, Kalsbeek A (2019) Circadian clocks and insulin resistance. Nat Rev Endocrinol 15(2):75-89. https://doi.org/10.1038/s41574-018-0122-1

32. Léger D, Debellemaniere E, Rabat A, Bayon V, Benchenane K, Chennaoui M (2018) Slow-wave sleep: from the cell to the clinic. Sleep Med Rev 41:113-132. https://doi.org/10.1016/j.smrv.2018. 01.008

33. Stamatakis KA, Punjabi NM, Stamatakis KA, Punjabi NM (2010) Effects of sleep fragmentation on glucose metabolism in normal subjects. Chest. 137(1):95-101. https://doi.org/10.1378/chest.090791

34. Van Cauter E, Leproult R, Plat L, Van Cauter E, Leproult R, Plat L (2000) Age-related changes in slow wave sleep and REM sleep and relationship with growth hormone and cortisol levels in healthy men. JAMA. 284(7):861-868. https://doi.org/10.1001/jama.284.7. 861

35. Møller N, Jørgensen JOL (2009) Effects of growth hormone on glucose, lipid, and protein metabolism in human subjects. Endocr Rev 30(2):152-177. https://doi.org/10.1210/er.2008-0027

36. Davidson JR, Moldofsky H, Lue FA (1991) Growth hormone and cortisol secretion in relation to sleep and wakefulness. J Psychiatry Neurosci 16(2):96-102

37. Spiegel K, Leproult R, Van Cauter E (1999) Impact of sleep debt on metabolic and endocrine function. Lancet. 354(9188):1435-1439. https://doi.org/10.1016/S0140-6736(99)01376-8

38. Keckeis M, Lattova Z, Maurovich-Horvat E et al (2010) Impaired glucose tolerance in sleep disorders. PLoS One 5(3):1-6

39. Briançon-Marjollet A, Weiszenstein M, Henri M, Thomas A, Godin-Ribuot D, Polak J (2015) The impact of sleep disorders on glucose metabolism: endocrine and molecular mechanisms. Diabetol Metab Syndr 7(1):1-16

Publisher's note Springer Nature remains neutral with regard to jurisdictional claims in published maps and institutional affiliations. 